\title{
Franc-maçonnerie et mouvement coopératif en région parisienne (1871-1914)
}

Freemasonry and the cooperative movement in the Paris region (1871-1914)

\section{Masonería y movimiento cooperativo en la región de Paris (1871-1914)}

\section{Eric Lebouteiller}

Numéro 341, juillet 2016

URI : https://id.erudit.org/iderudit/1037439ar

DOI : https://doi.org/10.7202/1037439ar

Aller au sommaire du numéro

Éditeur(s)

Association Recma

ISSN

1626-1682 (imprimé)

2261-2599 (numérique)

Découvrir la revue

Citer cet article

Lebouteiller, E. (2016). Franc-maçonnerie et mouvement coopératif en région parisienne (1871-1914). Revue internationale de l'économie sociale, (341),

104-117. https://doi.org/10.7202/1037439ar
Résumé de l'article

La franc-maçonnerie, dans la région parisienne, a entretenu, entre 1871 et 1914, des relations fluctuantes avec le mouvement coopératif. Toutefois, elle a participé manifestement, tant au niveau individuel que collectif, au renouveau du projet coopérateur après l'écrasement de la Commune. Même s'il est encore difficile d'évaluer avec précision le poids des Francs-maçons dans les milieux coopérateurs, il est en effet indéniable qu'un grand nombre de frères se sont intéressés autant aux pratiques coopératives qu’à la réflexion intellectuelle sur le sujet. Comme sociétés de pensée, les loges ont ainsi contribué par leurs travaux à promouvoir le mouvement coopérateur. 


\title{
FRANC-MAÇONNERIE ET MOUVEMENT COOPÉRATIF EN RÉGION PARISIENNE (1871-1914)
}

\author{
par Eric Lebouteiller*
}

La franc-maçonnerie, dans la région parisienne, a entretenu, entre 1871 et 1914, des relations fluctuantes avec le mouvement coopératif. Toutefois, elle a participémanifestement, tant au niveau individuel que collectif, au renouveau du projet coopérateur après l'écrasement de la Commune. Même s'il est encore difficile d'évaluer avec précision le poids des Francs-maçons dans les milieux coopérateurs, il est en effet indéniable qu'un grand nombre de frères se sont intéressés autant aux pratiques coopératives qu'à la réflexion intellectuellesur le sujet. Commesociétés de pensée, les loges ont ainsi contribuéparleurs travauxà promouvoirle mouvement coopérateur.

\section{Freemasonry and the cooperative movement} in the Paris region (1871-1914)

Freemasonry in the Paris region had a fluctuating relationship with the cooperative movement between 1871 and 1914 . However, freemasonry was clearly involved, at both the individual and collective level, in the revival of the cooperative movement after the suppression of the Commune. Even if it is hard to estimate precisely the number of freemasons in the cooperative movement, there was unquestionably a large number of freemasons who were interested in cooperative practices and ideas. As societies for intellectual discussions, the lodges helped foster the cooperative movement through their activities.

\begin{abstract}
Masonería y movimiento cooperativo en la región de Paris (1871-1914)

La masonería en la región de Paris ha manteniendo relaciones fluctuantes con el movimiento cooperativo entre 1871 y 1914. Sin embargo, ha participado obviamente, tanto al nivel individual que al colectivo, al renacimiento del proyecto cooperador después del aplastamiento de la Comuna. En efecto, si bien es aún difícil evaluar de manera precisa la influencia de los masones en los entornos cooperativos, es indudable que numerosos hermanos se interesaron tanto por las prácticas cooperativas como por la reflexión académica sobre el asunto. Como sociedades de pensamiento, las logias han contribuido a través de sus trabajos a promover el movimiento cooperativo.
\end{abstract}

* Bibliothécaire en université, historien du mouvement ouvrier et de la franc-maçonnerie au xixe siècle (membre de l’Iderm, Institut d'études et de recherches maçonniques).

A Anthony Lorry (1972-2014). 
(1) Assemblée législative annuelle composée de délégués des loges au Grand Orient de France.
A u convent de 1931, le responsable politique Paul Ramadier désignait la coopération comme "fille de la maçonnerie». Cette idée d'une parenté entre le projet coopérateur et l’idéal maçonnique est communément admise, mais la réalité est certainement plus complexe. La relation n'a-t-elle pas plutôt varié au cours du temps, entre proximité et distanciation? Le sujet a été jusqu'à présent peu traité. Les rares travaux qui l'ont abordé s'intéressent à l'ensemble de la question sociale. Aussi, l'étude qui s'appuie sur la consultation d'archives de loges, la lecture des comptes rendus de convents ${ }^{(1)}$ et de la presse maçonnique, entend évaluer la place de la franc-maçonnerie dans l'essor du mouvement coopératif, pour finalement se demander si elle ne constitue pas en son sein un milieu culturel original.

L'étude prend naturellement en compte les branches prospères de la coopération en milieu urbain, à savoir la production, la consommation et, partiellement, le crédit et le logement. Il est en revanche encore prématuré d'élargir le sujet à l'ensemble du territoire français. Limiter le champ d'étude à l'espace parisien et à sa proche banlieue est aussi nécessaire qu'approprié: laboratoire social et idéologique puissant, la région constituerait un modèle régional à part entière par son dynamisme et son rôle moteur. Présent sur l'ensemble d'un territoire fortement industrialisé, le mouvement coopératif parisien connaît cependant des «divisions graves» (Gaumont, 1924, p. 465).

A Paris, la coopération ouvrière domine dans les arrondissements périphériques, tandis que la coopération ménagère bénéficie du soutien des cercles conservateurs et libéraux. Dans les autres départements de la Seine, l'essor de la coopération est lié à la croissance industrielle de la banlieue et à son urbanisation récente. Parallèlement, la région est nettement structurée par deux grandes obédiences politisées, mais à l'évolution différente. Alors que le Grand Orient de France connaît une relative stabilité et se mue en foyer de propagation du laïcisme, le Rite écossais se divise. Le Suprême Conseil de France est confronté au schisme de loges avancées, qui créent en 1880 la Grande Loge symbolique écossaise. Ils se réconcilient en 1894 au sein de la Grande Loge de France.

En somme, les années 1870-1914 sont une période féconde pour l'observation des relations entre deux institutions phares sous la III ${ }^{e}$ République. L'évolution des rapports entre franc-maçonnerie et coopération peut être analysée en trois temps: après l'écrasement de la Commune, qui met fin à la brève expérience des premières coopératives modernes, la première phase est marquée par l'essor lent mais continu du mouvement coopératif; le soutien des Républicains est ensuite peu à peu concurrencé par l'affirmation du courant socialiste; l'entrée dans le $\mathrm{xx}^{\mathrm{e}}$ siècle est enfin une période d'intense réflexion maçonnique sur le projet coopérateur. 
(2) L. Martel crée La Solidarité (XIXe arrondissement), L. Delhomme L’Avenir de Plaisance (XIV) puis L’Avenir de Vaugirard $\left(\mathrm{XV}^{\mathrm{e}}\right)$.
(3) Synonyme de maçonnique (allusion au mythe d'Hiram, fondateur de la symbolique maçonnique).

\section{1-1885 : La renaissance du mouvement coopératif}

\section{Réseau maçon et renouveau du mouvement coopératif}

Malgré la répression versaillaise qui frappe le monde ouvrier parisien après l'échec de la Commune, le projet coopérateur renaît dans la région à partir des années 1873-1874. Quel rôle ont joué les Francs-maçons dans le renouveau du mouvement?

\section{LE POIDS DES COOPÉRATEURS MAÇONS}

Faute de travaux, il est prématuré d'évaluer précisément la présence franc-maçonne dans le milieu coopératif. L’examen des annuaires maçonniques permet toutefois de repérer les états-majors des sociétés, et non la base, encore difficile à identifier.

Portée par l'idéal démocratique et une aspiration à la solidarité, la maçonnerie parisienne est engagée, depuis la fin de l'Empire, dans le mouvement coopératif. Lors du premier Congrès ouvrier, à Paris en 1876, la moitié au moins des délégués des coopératives ouvrières sont initiés. Premières à renaître dans les quartiers populaires, les coopératives de consommation comptent précisément parmi leurs fondateurs, la plupart ouvriers de métier, de nombreux maçons actifs ${ }^{(2)}$. Le cas de L'Egalitaire, "coopérative alimentaire " fondée en 1876 dans le $\mathrm{X}^{\mathrm{e}}$ arrondissement (Calvarin, 2007), est aussi significatif, bien qu'apparemment isolé. Son comité d'initiative, issu des loges L’Avenir et Les Amis de la Tolérance, est clairement maçonnique. Cette filiation est perceptible jusque dans Les armes parlantes de la nouvelle société: équerre et fil à plomb (Gaumont, 1920, p. 5). A l'inverse, La Bellevilloise, née la même année de la volonté d'un groupe de mécaniciens du $\mathrm{XX}^{\mathrm{e}}$ arrondissement, paraît plus éloignée de ce milieu.

En parallèle, les acteurs de la coopération de production, proches de l'esprit quarante-huitard, sont certainement plus "maçonnisés ». Le lien ancien entre idéaux associationniste et maçonnique, transmis par les vétérans du mouvement, tel l'ouvrier A. Cohadon, se perpétue dans les sociétés parisiennes, par exemple à l'Association des tailleurs d'habits, gérée par les Communards V. Godfrin et E. Evette, ou à l'Imprimerie nouvelle, dirigée par G. Masquin. Le renouveau tardif du mouvement, dans les années 1880 , révèle toutefois une nouvelle génération de dirigeants, autour de la Chambre consultative des associations ouvrières de production, à la forte présence hiramiste ${ }^{(3)}$. Les premiers administrateurs sont maçons, le fouriériste H. Buisson, le papetier A. Vila ou encore A. Romanet, responsable de la Lithographie parisienne. En banlieue, le mouvement se structure aussi autour de figures très souvent franc-maçonnes. R. Steinmetz, 
(4) Le Rappel, A. Vacquerie (dir.); La Tribune, F. X. Trébois (dir.).

(5) Dirigeant du Grand Orient de France.

(6) Circulaire du grand maître Babaud-Laribière, Le Monde maçonnique, juin-juillet 1870, p.138. fondateur à Pantin de deux sociétés, lance avec H. Vaudémont, coopérateur à Joinville, L'Association fraternelle, organe des sociétés coopératives de la région. Des loges sont parfois même à l'initiative. Les Rénovateurs, atelier socialiste de Clichy, fournit ainsi en 1885 «le noyau de base de la coopérative L'Economie sociale» (Lauzeray, 1987, p. 77).

\section{LA CONSTITUTION D'UN GROUPE D'INFLUENCE}

En vue de former un parti "coopératiste", le mouvement encourage l'activité de groupes d'études sociales, principalement implantés à Paris. En 1872 est fondée la Société d'études pratiques pour le développement des associations coopératives. Les fondateurs maçons sont des vieux militants ouvriers (A. Cohadon, M. Nadaud, H. Tolain...). Ce cercle de réflexion, qui plaide pour la coopération de production, est soutenu par Le Rappel et La Tribune, journaux républicains, qui publient, sous la houlette d'initiés notoires ${ }^{(4)}$, une rubrique coopérative lue dans le monde ouvrier.

De son côté, la presse associationniste renaît souvent grâce à l'appui maçonnique. Le Bulletin (puis Revue) du mouvement social (1872-1887), qui diffuse la pensée fouriériste influente chez les coopérateurs, est tenu par les frères $\mathrm{Ch}$. Limousin et $\mathrm{E}$. Nus. Plus tard, la Société républicaine d'économie sociale est une émanation de La Revue socialiste. Liés au monde maçonnique, ses fondateurs (B. Malon, Z. Camélinat, E. May) défendent la coopération ouvrière, qu'ils espèrent promouvoir à l'Exposition universelle de 1889 à Paris, mais le projet avorte avec la dissolution de la société lors de la crise boulangiste.

\section{L'action coopérative des loges}

\section{LA CAUSE RÉFORMISTE}

La décennie qui suit la répression de la Commune est caractérisée dans les obédiences par l'ouverture aux débats de société. Au Grand Orient, Babaud-Laribière, premier grand maître républicain ${ }^{(5)}$, donne l'impulsion dans une circulaire de 1870 : "Il me paraît urgent d'aborder l'étude des questions sociales. Telle Loge est bien placée [...] pour établir une bonne harmonie entre patrons et ouvriers; telle autre s'occupera avec fruit de l'organisation des sociétés coopératives ${ }^{\left({ }^{(6)}\right.}$ ». L’initiative s'appuie sur l'action d'un courant réformateur, né dans l'opposition à l'Empire. Tenant d'une République démocratique, égalitaire et laïque, il contribue à relancer l'idée coopérative dans les loges. Pour Le Monde maçonnique, la grande revue réformiste, l'étude des transformations sociales doit cependant être mesurée: "Les Loges peuvent sans danger aborder ces graves et difficiles questions en s'inspirant des sentiments maçonniques, agissant sans parti pris et sans passion, dans le seul intérêt du bien-être général» (Caubet, 1879). 
Finalement, la promotion de l'utopie associative dans les loges est née de la rencontre entre une maçonnerie libérale et les dirigeants du mouvement ouvrier initié dans les années 1860. Au Grand Orient, Les Amis de la tolérance au recrutement populaire se déclarent dès le début, en 1869, "partisans des coopératives qui bien menées doivent affranchir l'ouvrier» (Combes, 1978, p. 17). Autre foyer politique plus lettré, La Renaissance propose «une étude sur l'importance sociale des Congrès ouvriers » (Caubet, 1878), encore enclins à la coopération. De même, parmi les loges écossaises avancées, La Justice et La Prévoyance sont présidées par l'avocat P. Goumain-Cornille et le militant A. Bibal, des promoteurs des coopératives de crédit.

\section{LE CHAMP D'ACTION DE LA FRANC-MAÇONNERIE}

La franc-maçonnerie n'est pas seulement un lieu de réflexion, elle s'investit aussi dans la réalisation d'expériences pratiques. Dans le cadre de ses activités philanthropiques, l'Ordre entreprend ainsi, en matière de coopération, une action éducative, afin d'encourager la lecture dans les milieux populaires. Un réseau modeste de bibliothèques coopératives se constitue dans les années 1870 dans le nord et l'est de la capitale, sous le patronage de figures de la maçonnerie parisienne, telles que H. Harant, E. Lefebvre ou A. Desmoulins. Par son action pionnière, le mouvement contribue à l'émergence de la coopération scolaire de la fin de siècle.

Mais le domaine d'intervention de la maçonnerie est sans conteste le financement de la coopération. Face au manque de capitaux suffisants, qui a freiné le développement du réseau, l'apport financier, plutôt discret, des loges a manifestement permis la création de sociétés coopératives (Chevallier, 1975, p. 132). A Paris, d'après les travaux de J. Gaumont (1920, 1932), seule l'Egalitaire semble avoir bénéficié d'un soutien maçonnique clair. Enfin, une large réflexion est menée dans les loges en faveur de l'organisation du crédit. Des brochures sont parfois éditées, telle la planche ${ }^{(7)}$ de $\mathrm{J}$. Darmoy à La Constante

(7) Discours prononcé dans une loge par un frère sur un fait d'actualité ou sur la symbolique.

(8) Parmi les délégués, plusieurs coopérateurs maçons (Ch. Chabert, J. Dupire, L. Delhomme).

(9) Le terme « vénérable » désigne le président d'une loge maçonnique.

(10) Brochure de L’Etoile polaire sur l'historique de la loge (1939), p. 35. Amitié (Darmoy, 1885). Surtout, des projets de banque associative destinés à aider la coopération de production émergent dans les obédiences. En 1873, la délégation ouvrière ${ }^{(8)}$ à l'Exposition universelle de Vienne (Autriche) propose dans son rapport la création d'un crédit mutuel coopératif, afin de financer les sociétés ouvrières (Caubet, 1873). En 1876, l'appel relayé par la maçonnerie autour des vénérables ${ }^{(9)} \mathrm{A}$. Martin et $\mathrm{A}$. Bibal, " en vue de la fondation d'une banque populaire coopérative» (Gaumont, 1924, t. 2, p. 52) sur le modèle du réseau bancaire transalpin, n'aboutit pas. En revanche, S. de Heredia, de L'Etoile polaire, suite à une conférence du frère F. Vigano, promoteur du crédit en Italie, réunit un groupe d'hommes appartenant aux deux obédiences (M. Nadaud, E. Benoît-Lévy, D. Poulot...) et obtient l'autorisation de créer en 1880 la Caisse centrale du travail et de l'épargne ${ }^{(10)}$. 
Les Francs-maçons ont véritablement joué durant cette période un rôle déterminant dans la lente reconstitution des branches de la coopération.

\section{5-1900 : Le mouvement républicain face à la percée socialiste}

\section{Le patronage républicain}

L'accession des Républicains au pouvoir permet de consolider le développement du mouvement coopératif dans son ensemble.

\section{LE DÉBAT PARLEMENTAIRE}

Avant même l'instauration en 1912 du Groupe parlementaire de la coopération, un noyau d'élus spécialisés se retrouve lors des discussions sur l'économie sociale au Sénat et à la Chambre des députés, centres du pouvoir politique depuis les lois constitutionnelles de 1875. Au cœur du processus d'élaboration législative, le travail parlementaire devient assurément l'un des lieux de maturation de l'idée coopérative. Dans l'hémicycle, le débat en séance est le moment privilégié de confrontation des différentes sensibilités. Pour autant, ce groupe informel partage globalement le projet républicain d'encadrer la coopération par la loi. En partie lié par un engagement maçonnique commun, il réunit dans les années 1880-1890, autour des Républicains H. Maze et V. Lourties, plus préoccupés par la mutualité, les élus ouvriers H. Tolain et $M$. Nadaud, les chefs radicaux Eugène et Camille Pelletan, les conservateurs sociaux Jules Simon et Léon Say, favorables à la coopération de crédit.

\section{LE PROJET LÉGISLATIF}

A la tête de l'Etat républicain, des dirigeants engagés en maçonnerie œuvrent au progrès du projet coopérateur en France. Dignitaire de la loge écossaise La Justice, Charles Floquet en est un fervent partisan. Préfet de la Seine en 1882, il organise pour Paris la gestion du legs Rampal, destiné à accorder des prêts aux coopératives, puis leur ouvre le marché des travaux de la ville (Gueslin, 1998, p. 316). Chef du gouvernement en 1888, il leur octroie d'autres dispositions favorables, le droit de préférence et la dispense de cautionnement en cas de participation aux marchés de l'Etat.

Charles Floquet dépose aussi au Parlement, en 1888, un projet de loi ambitieux, résultat de la grande enquête de 1883 dans les milieux coopératifs. Il s'agit de réformer la loi partielle de 1867 sur les sociétés ouvrières en octroyant un statut autonome à la coopération dans le cadre d'une législation unitaire. La

(11) Instance dirigeante du Grand Orient de France, présidé par le grand maître. commission en charge du projet révèle une figure politique montante, le député Paul Doumer (Lorin, 2013). Le rapporteur de la loi, jeune élu au Conseil de l'ordre ${ }^{(11)}$, se fait défenseur 
(12) Anonyme, 1889, «L'Ecole mutuelle: les sociétés coopératives ouvrières de consommation et de production ", La Chaîne d'union, janvier, p. 7-8.

\footnotetext{
(13) Notamment à « La France maçonnique ", conférence sur le livre de L. Bourgeois, Bulletin hebdomadaire des travaux de la maçonnerie en France, janv. 1897, p. 3.
}

de la coopération dans les milieux maçonniques. Il planche ainsi à L'Ecole mutuelle devant un aréopage de dignitaires maçons ${ }^{(12)}$. L'échec de la loi, après dix ans de bataille parlementaire n'afflige pourtant pas le monde coopératif. Son principal théoricien, Charles Gide, assène: "La coopération est née sans qu'aucun législateur l'ait baptisée» (1896, p. 37).

\section{LA PROMOTION DU SOLIDARISME}

Proche de Charles Floquet, Léon Bourgeois est un membre éminent du Grand Orient, très attaché aux idéaux de justice sociale. Président du Conseil en 1895, il constitue le premier gouvernement radical, véritable «ministère des Loges » avec sept Maçons. La brièveté de l'expérience l'amène à repenser son action dans un essai politique. Paru en 1896, l'ouvrage Solidarité expose la doctrine solidariste, à mi-chemin entre libéralisme et collectivisme. Sa pensée, fondée sur le principe de solidarité et sur la généralisation de l'association, apparaît " comme la conceptualisation des pratiques coopératives et mutualistes 》 (Dreyfus, 2013, p. 36). Une série de planches est donnée dans les loges ${ }^{(13)}$ pour promouvoir l'ouvrage en passe de devenir la doctrine sociale et économique du radicalisme. De surcroît, des loges d'essence solidariste ouvrent. En 1904, sont créées, à Paris, La Solidarité, par le syndicaliste V. Dalle, et Travail et solidarité, animée à Auteuil par R. Coupot, sociétaire de l'Abeille de Passy.

En septembre 1890, les trois législateurs de la coopération, Floquet, Doumer et Bourgeois fondent Voltaire, un atelier qui se veut un haut lieu de réflexion. Cet élitisme républicain va cependant à l'encontre du changement qui s'opère dans la composition sociologique des loges.

\section{L'entrée en masse des socialistes en franc-maçonnerie}

\section{LA CONSTITUTION D'UN RÉSEAU DE LOGES SOCIALISTES}

Le recrutement maçonnique des milieux ouvriers, entériné au Grand Orient en 1893 par la baisse des cotisations, a repris après la loi d'amnistie de 1880 et le retour dans les temples des survivants de la Commune. Maints militants de la coopération renouent ainsi avec la maçonnerie nationale. Beaucoup rejoignent en particulier les loges avancées du Rite écossais. L’ancien dirigeant A. Avrial et le gérant de la Société de Picpus, R. Urbain, s'affilient à La Philosophie sociale, une loge anarchisante, tandis que C. Langevin, organisateur de coopératives de production, s’inscrit à La Prévoyance, vieil atelier coopérativiste. Au Grand Orient, Le Lien des peuples accueille E. Fournière et B. Malon, dont la pensée intègre largement la dimension coopérative. Plus tardivement, la loge de Clichy, Les Rénovateurs (Lauzeray, 1987), initie en 1898 le poète militant J.-B. Clément puis J. Allemane, gérant d'une imprimerie coopérative. 
(14) Anonyme, 1891, « Travaux des ateliers franc-maçonniques ", Bulletin maçonnique, mai, p. 288-289.

(15) Déclaration du 20 février 1893 (Annuaire de la loge, 1896).
(16) Anonyme, 1890, « Conseil de l'ordre », Bulletin du GOF, janvier, t. 45 , p. 559.
(17) Assemblée générale (AG) de 1894, "Programme d'études ", Bulletin du GOF, t. L, p.112-117.

(18) Réunions de travail des loges écossaises, selon un rituel préétabli. (19) Anonyme, 1888, « Chroniques des travaux des ateliers francmaçonniques ", La Chaîned'union, juillet, p. 268-279.

(20) La Sociale, fondée à Montmartre en 1882 .
Le retour progressif des ouvriers en maçonnerie encourage alors un mouvement de création de loges à vocation sociale. A l'origine boulangiste, Thélème se recentre sur les thèmes socio-économiques (Rouault, 2014), recrute des coopérateurs et propose en 1891 une série de planches sur l'histoire du mouvement ${ }^{(14)}$. Parallèlement, au Rite écossais, l'atelier parisien Lutecia insère la coopération dans son programme d'études sociales (Gourdot, 1999, p. 239). Cet engagement se traduit parfois par l'adhésion au socialisme. L'Ecole mutuelle, animée par G. Francolin, adopte ainsi le programme du Parti ouvrier, d'inspiration guesdiste ${ }^{(15)}$. Les socialistes restent néanmoins dispersés dans des ateliers à majorité radicale, aussi décident-ils d'ouvrir leurs propres loges, plus aptes à recevoir des coopérateurs. Les Hospitaliers socialistes, au recrutement ouvrier, accueillent de jeunes praticiens, tels que L. Deslinières et Fr. Brunet. Ce dernier fondera en 1904 avec P. Brousse Les Travailleurs socialistes de France (Combes, 1999, p. 292), atelier fréquenté par des dirigeants (V. Voinot, H. Bagnol). L'apogée de ce réseau sera atteint dans les obédiences avant 1914. Il renforce l'influence socialiste, qui domine depuis 1892 les congrès des loges de la région parisienne et finit par peser sur l'orientation générale des convents.

\section{LE PROGRAMME D'ÉTUDES SOCIALES}

Le regain d'intérêt pour l'étude des transformations sociales pousse les instances dirigeantes des grandes obédiences à intervenir. Au Grand Orient, le Conseil de l'ordre adopte en 1890 la proposition de Francolin, qui vise à examiner les problèmes socio-économiques ${ }^{(16)}$. De même, à la Grande Loge symbolique écossaise, la revue dirigée par P. Goumain-Cornille (1890) déclare: «La Franc-maçonnerie doit s’investir dansle mouvement social. » De fait, les fédérations maçonniques semblent encore plus favorables au "développement du mouvement coopératifet de la participation aux bénéfices, considérés comme deux étapes nécessaires de l'évolution sociale ${ }^{(17)} 》$.

Dans les loges écossaises, malgré la scission de 1880 qui oppose la maison mère à une jeune obédience plus progressiste, l'aspiration au progrès social semble un combat commun. Au Suprême Conseil, les tenues plénières ${ }^{(18)}$ de 1887 et 1888 consacrées au travail abordent la participation aux bénéfices et la coopération, sujet de controverse après les propos en séance du docteur Gonnard en $1888^{(19)}$. De son côté, l'aile gauche du Rite écossais, dirigée par une élite radicale, procède à l'installation de loges à vocation sociale ${ }^{(20)}$ et poursuit son action en faveur de l'organisation du crédit. Enfin, des ateliers proposent en 1893 une série de débats d'essence socialiste, intitulée " $L a$ Franc-maçonnerie, organisatrice du prolétariat " (Combes, 1999, p. 193). 
(21) Anonyme, 1889, «Chronique des travaux des ateliers franc-maçonniques ", La Chaîne d'union, janvier, p. 5-8.

(22) Anonyme, 1893, " Groupe d'études des loges de la région parisienne ", Bulletin maçonnique, mai, p. 123-124.

(23) AG de 1896, "Questions renvoyées à l'étude des loges », Compte rendu des travaux du GOF, t. LII, p. 338-339.

(24) La campagne est aussi l'occasion d'études dans les loges (L’Union des peuples, Les Héros de l'humanité...), Bulletin hebdomadaire des travaux de la maçonnerie en France, janvier-avril 1896.

(25) Anonyme, 1896, «Comité central de l'Union coopérative ", in L'Almanach de la coopération française (page non numérotée).
Au Grand Orient, les loges multiplient les discussions contradictoires afin d'encadrer, selon un rituel strict, les débats plus controversés. En 1889, l'exposé de L. Lagasse aux Admirateurs de l'univers sur "les avantages et les inconvénients des sociétés coopératives de production, de crédit et de consommation » est contesté par des frères ${ }^{(21)}$. Par ailleurs, les loges de la région parisienne constituent en 1893 un groupe de travail, avec Benoît-Lévy et Francolin, qui inclut dans le plan d'étude la participation aux bénéfices et les sociétés coopératives ${ }^{(22)}$. Le temps fort reste toutefois le convent social organisé en 1896. Faute de temps, les propositions sur la participation et «la question de la propriété et de ses différentes formes sociales ${ }^{(23)}$ » sont renvoyées à l'étude des loges, sous l'autorité de la Commission des études sociales.

A la fin du siècle, le courant socialiste s'est durablement implanté en maçonnerie. Il s'est structuré autour d'actions collectives. En 1895, la vaste campagne de soutien à la coopérative des verriers d'Albi mobilise l'aide financière d'ateliers parisiens notoires ${ }^{(24)}$.

\section{0-1914 : La franc-maçonnerie au cœur de la réflexion}

\section{Les limites du projet coopératif}

Alors que le milieu de la coopération de production reste fortement "maçonnisé », les sociétés de consommation traversent dans les années 1900 une crise profonde.

\section{UN MODĖLE COOPÉRATIF EN CRISE}

Au tournant du siècle, le mouvement coopératif de consommation, pourtant en plein essor, se divise. Les coopérateurs socialistes quittent l'Union coopérative pour fonder, en 1895, la Bourse des coopératives socialistes. Dans les deux fédérations, la présence maçonnique, difficile à évaluer, semble en recul par rapport à la période précédente.

Du côté des partisans d'une coopération neutre, les initiés sont visiblement en minorité. Le Comité central de la rue Christine ne compte en 1895 qu'un tiers de maçons en activité(25). Des liens étroits existent néanmoins. L’influente Société civile du XVIII ${ }^{e}$ arrondissement organise sa fête familiale au siège du Grand Orient, rue Cadet (Soria, 1896). Quelques personnalités, telles L. Mabilleau ou M. Dufourmantelle, sont des hommes clés de la coopération de crédit et d'habitation. Charles Gide, lui, est profane, mais ses jeunes disciples (A. Daudé-Bancel, R. Picard, G. Fauquet) ont intégré des loges. En banlieue, l'influence paraît plus marquée, car liée à des figures locales: E. Thomas à Gentilly, E. Kugler à Pantin. A la Bourse fondée par les grandes coopératives parisiennes, la présence hiramiste 
(26) Démissions de L. Lucas de L'Education coopérative ou de Ch. Mutschler du Niveau social, à Saint-Mandé (cf. fichier de Vichymis en place par le régime de Vichy afin d'identifier les Francs-maçons, ce fichier aujourd'hui informatisé est consultable en bibliothèque au GOF et à la GLDF).

(27) Union coopérative : A. DaudéBancel, A. Nast ; Bourse des coopératives socialistes : E. Poisson, L. Lucas, H. Sellier, P. Waseige. est forte dans le courant réformiste. Le réseau constitué par Albert Thomas comprend des coopérateurs maçons comme lui : E. Poisson, H. Sellier ou le jeune P. Ramadier. De même, en région parisienne, des acteurs locaux s'imposent: L. Voilin à Puteaux ou Th. Béguin à Ermont.

En outre, au début du siècle, un scandale frauduleux de malversations touche les principales sociétés parisiennes (L’Egalitaire, L’Avenir de Plaisance, La Moissonneuse). Parmi les coopérateurs qui conduisent le redressement moral et financier, se signalent des Maçons enclins par principe à la probité. En 1912, E. Poisson plaide pour l'avènement d'une "minorité consciente» au sein de cercles ouverts aux seuls militants afin d'"attirer et grouper les éléments sains " de la coopération ouvrière (Poisson, 1912). A la Bellevilloise, le puissant Cercle des coopérateurs $\mathrm{du} \mathrm{XX}^{\mathrm{e}}$ arrondissement agit sous l'impulsion de J. Lauche, affilié à L'Action socialiste. Surtout, la formation, sur le modèle anglais, d'un Magasin de gros (Gueslin, 1998, p. 299), centrale d'achats que gèrent les frères G. Lebon ou P. Waseige, est née de la transformation en 1906 de la Fédération parisienne animée par A. Dherbécourt, de la loge socialiste La Raison.

\section{LA MONTÉE DE L'ANTI-MAÇONNISME}

Contrairement au milieu de la coopération de production, toujours lié à l'Ordre, le mouvement coopératif de consommation connaît, à l'instar de la sphère syndicale et politique, une vague de contestation anti-maçonnique. L'affaire " des Fiches ", qui secoue le Grand Orient en 1904, déclenche la virulence des critiques de gauche contre "cette confrérie du mardi gras, figée dans des rites caducs » (Janvion, 1912, p. 7). Un climat de défiance gagne donc un monde jusqu'à présent bien disposé. Dans les loges, les démissions de coopérateurs se multiplient, signe d'un désamour grandissant ${ }^{(26)}$. Le courant hostile, ancien dans le milieu avancé des dirigeants parisiens (A. Hamelin, X. Guillemin), perdure avec la génération d'administrateurs post-dreyfusienne (L. Héliès, E. Buguet, A. Henriet...). Adeptes d'un socialisme révolutionnaire, ces coopérateurs non maçons fustigent un Ordre dominé socialement. Parmi eux, Marcel Mauss, taxé un jour de coopérateur bourgeois, se demande si «les véritables bourgeois ne sont pas les candidats politiques louches, les suppôts de Millerand ou les membres d'une société secrète éminemment bourgeoise, la franc-maçonnerie» (Fournier, 1995).

De fait, la perte d'influence maçonnique dans le mouvement coopératif de consommation paraît évidente. Lors du Pacte d'unité de 1912, on ne relève que six frères parmi les quatorze signataires ${ }^{(27)}$ et plus que quatre sur les vingt et un élus du premier comité confédéral de la FNCC. Les francs-maçons E. Poisson et A. Daudé-Bancel sont toutefois désignés secrétaires généraux. 
(28) Notices des membres de la loge Le Progrès, cf. fichier de Vichy (Archives du GOF).

(29) Anonyme, 1904, «Travaux du Conseil de l'Ordre ", Compte rendu des travaux du GOF, t. LX, janv.déc., p. 9-10.

(30) Lettre du 24 avril 1912, boîte d'archives $n^{\circ} 147$ (Archives du GOF). (31) Livre d'architecture de la loge (juillet 1910), p. 18-22 (Archives GDLF).

(32) AG de 1904, " Rapport sur la question du capital et du travail ", Compte rendu des travaux du GOF, t. LX, p. 263.

\section{Un engagement maçonnique fort}

\section{L'ORGANISATION DU DÉBAT}

Au début du siècle, apparaissent de nouveaux ateliers, dont la titulature reflète les convictions coopérativistes. Déjà instigateur de la Banque coopérative, un groupe de directeurs d'associations ouvrières du bâtiment, mené par H. Buisson et L. Favaron, ouvre en 1904 L'Education coopérative, née d'une scission à la loge radicale Le Progrès ${ }^{(28)}$. D'emblée, ils "se proposent de faire dans les loges une campagne en faveur de la coopération de production, qui résout une partie de la question sociale en supprimant le patronat ${ }^{(29)}$ ». D'autres ateliers au titre explicite s'engagent. L'Economie sociale émet en 1912 un vœu relatif à "la construction d'habitations salubres pour la classe ouvrière $^{(30)}$ " tandis que l'orateur de La République sociale, fondée par des employés des Postes "estime que la coopération seule permettra de solutionner la question sociale, et que c'est de ce côté, abstraction faite de vaines théories, que doivent tendre tous les efforts du prolétariat ${ }^{(31)}$.

\section{LES THĖMES DU DÉBAT}

Dans les convents du Grand Orient avant 1914, la réforme sociale est au cœur de la réflexion maçonnique et l'organisation du rapport entre capital et travail reste un débat majeur. Le conflit, entre les Radicaux et les Socialistes qui dominent les assemblées, porte sur des conceptions opposées. Les uns voient dans le projet coopératif un outil de paix sociale et de promotion individuelle dans le monde ouvrier. Les autres le considèrent, au contraire, comme moyen d'émancipation, apte à remplacer le salariat. L'association semble alors un remède à la question sociale. A. Bachelet de L'Etoile polaire, l'atteste: "La coopération, sous ses diverses formes, est appelée à jouer un grand rôle dans l'avenir ${ }^{(32)}$. " Souvent mis à l'étude, le sujet est inclus aux "Cahiers économiques et sociaux de la démocratie du XX ${ }^{\mathrm{e}}$ siècle », temps fort de l'activité des loges en 1905-1906. Son champ d'action est même étendu. En 1908, le débat porte sur l'organisation des services publics sur des bases coopératives et en 1909, sur la remise des concessions nouvelles (houille, soussol) aux seules sociétés ouvrières (Combes, 1999, p. 333-334). Ces propositions ambitieuses sont loin d'aboutir.

Une inflexion s'amorce cependant, que l'étude d'A. Sicard situe précisément. "Objet de tous les espoirs pendant dix ans, la coopération paraît subir au Convent de 1908 une bien triste défaite " (Sicard, 1994, p. 95). L'enquête menée alors auprès des loges révèle une forte minorité (trente-cinq loges) qui estime la coopération impuissante à remplacer le régime du salariat. L'autre débat s'articule sur la rivalité entre les modèles coopératifs de consommation et de production. Le radical 
(33) AG de 1904, "Coopération ", Compte rendu des travaux du GOF, t. LX, p. 347.

(34) Ibid., AG de 1906, p. 245.

(35) Ibid., AG de 1903, p. 191.
Ch.Debierre de L'Ecole mutuelle considère que « la coopération de consommation serait l'idéal car elle contient le moyen de créer et de développer la coopération de production en lui fournissant le capital $^{(33)}$ ». A l'inverse, d'autres la fustigent. Le député E. Chauvin la juge en 1906 « inutile et condamnée à végéter lorsque [...] elle cherche simplement à répartir en fin d'année un tant pour cent au prorata des cotisations $\left.{ }^{(34)}\right)$. Il est vrai que les républicains privilégient l'association ouvrière, objet de plusieurs résolutions en convent. L'une invite les ouvriers à créer " partout où c'est possible des coopératives de production qui leur permettent d'être les copropriétaires des instruments de production ${ }^{(35)}$ ».

De son côté, la Grande Loge de France, née de la réunification de la famille écossaise en 1894, se révèle progressiste sous l'égide du grand maître G. Mesureur. Les convents à l'orientation clairement sociale abordent les mêmes thèmes que le Grand Orient. L'étude des relations entre capital et travail est au programme en 1908. En 1913, la question de la transformation des moyens de production et d'échanges est renvoyée à l'étude des loges (Combes, 1999, p. 363-364). Les ateliers, tant socialistes que radicaux, proposent des travaux largement consacrés au progrès social. L'extrême politisation des loges est cependant peu à peu contestée par un courant symboliste, influent dans la Grande Loge.

Autour du projet coopérateur est ainsi apparu un œcuménisme républicain, qui s’inscrit dans la tentative de définir un socialisme maçonnique.

\section{Conclusion}

Au terme de ce travail à partir de l'étude des réseaux maçonniques et coopératifs, il apparaît, au cours de la séquence chronologique 1871-1914, que, si la franc-maçonnerie et la coopération, deux entités à leur apogée, ont été proches, elles n'ont jamais pu s’interpénétrer totalement. L'Ordre a certes été un acteur privilégié de l'essor du mouvement coopératif. Proche du mouvement ouvrier, son aile réformiste, séduite par les principes de fraternité et de solidarité inculqués par l'associationnisme, a eu, depuis les années 1860, un rôle moteur; mais l'engagement maçonnique en faveur de la coopération a été autant individuel que collectif. D'une part, les loges ont pu localement former l'ossature du mouvement coopératif en région parisienne. C'est particulièrement vrai en banlieue. Comme sociétés de pensée, elles ont aussi apporté une contribution importante à l'élaboration du projet en s'inspirant de l'idéal maçonnique. D’autre part, les militants maçons au sein du monde coopératif (A. Murat, H. Buisson, E. Poisson, etc.) ont pu s'apparenter à une minorité agissante. Ils ont été tour à tour propagandistes, praticiens ou législateurs de la coopération. Un changement notable 
(36) « Rouen et sa région, un berceau del'économie sociale et solidaire?», Colloque international d'histoire, Rouen, 2 et 3 octobre 2014. s’est néanmoins opéré dans les obédiences. Au fil du temps, les intervenants dans les convents ne sont plus des praticiens de la coopération comme auparavant, mais simplement des défenseurs convaincus de l'utopie ouvrière.

La culture maçonnique a également varié selon les familles de la coopération. Davantage liés au milieu républicain, surtout depuis le rejet du projet coopérateur par les congrès ouvriers après 1878 , les sociétaires d'associations ouvrières sont par tradition davantage maçons, dans l'esprit quarante-huitard. A l'inverse, la fréquentation des loges est plus contrastée parmi les coopérateurs de consommation. Autant la maçonnerie a joué un rôle décisif dans le renouveau du mouvement coopératif, autant, au tournant de 1900, la défiance est grandissante, avec la montée de l'antimaçonnisme. L’influence du socialisme révolutionnaire dans le milieu ouvrier parisien a sans doute contribué au rejet de l'Ordre.

Au regard de cette première synthèse loin d'être exhaustive, il serait intéressant de l'enrichir à terme, par le biais méthodologique de l'histoire comparée. Les régions de Nîmes et du Jura, à l'origine de modèles coopératifs originaux (Toucas-Truyen, 2005, p. 50-53), ont en effet développé chacune un lien particulier avec la franc-maçonnerie locale. Une analyse comparée pourrait permettre de spécifier les différentes situations, notamment autour de l'hypothèse féconde des « districts de l'économie sociale et solidaire ", notion au cœur d'un récent colloque ${ }^{(36)}$. 


\section{BIBLIOGRAPHIE}

Calvarin Cl., 2007, « L'Egalitaire, histoire singulière d'une utopie ouvrière ", Bulletin de la société historique $d u X^{e}$ arrondissement de Paris, numéro spécial.

Caubet J.-M., 1873, « La Justice, rite écossais: rapport sur l'exposition de Vienne, par le frère Godfrin ", Le Monde maçonnique, novembre, p. 321 .

Caubet J.-M., 1878, "Chronique Paris et départements ", Le Monde maçonnique, avril, p. 493.

Chevallier P., 1975, Histoire de la francmaçonnerie française, t. 3 (1877-1944), Paris, Fayard.

Combes A., 1978, « Un atelier d'avant-garde: Les Amis de la tolérance (1868-1883)", Chroniques d'histoire maçonnique, $\mathrm{n}^{\circ} 20$, p. 16-25.

Combes A., 1999, Histoire de la francmaçonnerie au XIX $X^{e}$ siècle, t. 2, Monaco, éd. du Rocher.

Darmoy J., 1885, Rapports démocratiques $d u$ travail et du capital. Le crédit démocratique, les banques populaires et les associations ouvrières, Paris, éd. La Constante amitié.

Dreyfus M., 2013, Financer les utopies. Une histoire du Crédit coopératif (1893-2013), Paris, Actes Sud.

Fournier M., 1995, Marcel Mauss, Paris, Fayard. Gaumont J.,1920, Les sociétés de consommation à Paris, Paris, Fédération des coopératives de la région parisienne.

Gaumont J.,1924, Histoire générale de la coopération en France, t. 1-2, Paris, FNCC.
Gaumont J., 1932, Les mouvements de la coopération ouvrière dans les banlieues parisiennes, Paris, PUF.

Gide Ch., 1896, " Projet de loi coopératif » in Almanach de la coopération française, Paris, comité central de l'Union coopérative.

Goumain-Cornille P., avril 1890, Bulletin maçonnique de la GLSE, p. 5.

Gourdot P., 1999, Le combat social des Francsmaçons, Monaco, éd. du Rocher.

Gueslin A., 1998 (rééd.), L'invention de l'économie sociale, Paris, Economica.

Janvion E., 1912, « La Franc-maçonnerie et la classe ouvrière ", conférence donnée à l'Hôtel des sociétés savantes.

Lauzeray C., 1987, « Clichy: maçonnerie et vie municipale ", Chroniques d'histoire maçonnique, $\mathrm{n}^{\circ} 39$, p. 71-84.

Lorin A., 2013, « La Franc-maçonnerie sous la III République: Paul Doumer (1857-1932) et le GOF ", Chroniques d'histoire maçonnique, $\mathrm{n}^{\circ} 71$, p. 50-66.

Poisson E., 1912, Le rôle des cercles coopératifs, FNCC.

Rouault T., 2014, « Thélème: histoire d'une loge atypique du GOF", Chroniques d'histoire maçonnique, $\mathrm{n}^{\circ}$ 73, p. 63-72.

Sicard A., 1994, "La question sociale au GOF (1893-1914) », mémoire de maîtrise, Paris Sorbonne.

Soria L., 1896, "Fête coopérative », l’Union coopérative, ${ }^{\circ} 18$, p. 281.

Toucas-Truyen P., 2005, Dreyfus M. (dir.), Les Coopérateurs, Paris, éd. de l'Atelier. 